\title{
Circuit
}

Musiques contemporaines

\section{Acoustique et forme chez Varèse}

\section{Gilles Tremblay}

Volume 6, numéro 1, 1995

Tremblay/Varèse/Messiaen : Gilles Tremblay analyste

URI : https://id.erudit.org/iderudit/902118ar

DOI : https://doi.org/10.7202/902118ar

Aller au sommaire du numéro

Éditeur(s)

Les Presses de l'Université de Montréal

ISSN

1183-1693 (imprimé)

1488-9692 (numérique)

Découvrir la revue

Citer cet article

Tremblay, G. (1995). Acoustique et forme chez Varèse. Circuit, 6(1), 23-36. https://doi.org/10.7202/902118ar

Ce document est protégé par la loi sur le droit d'auteur. L'utilisation des services d'Érudit (y compris la reproduction) est assujettie à sa politique d'utilisation que vous pouvez consulter en ligne.

https://apropos.erudit.org/fr/usagers/politique-dutilisation/
Cet article est diffusé et préservé par Érudit.

Érudit est un consortium interuniversitaire sans but lucratif composé de l’Université de Montréal, l'Université Laval et l'Université du Québec à Montréal. Il a pour mission la promotion et la valorisation de la recherche. https://www.erudit.org/fr/ 


\section{Acoustique et forme chez Varèse}

Article paru dans La Revue musicale, $n^{\circ} 383-385,1985$, pp. 29-46.

Que se passe-til lorsque je joue ceci :

Exemple 1

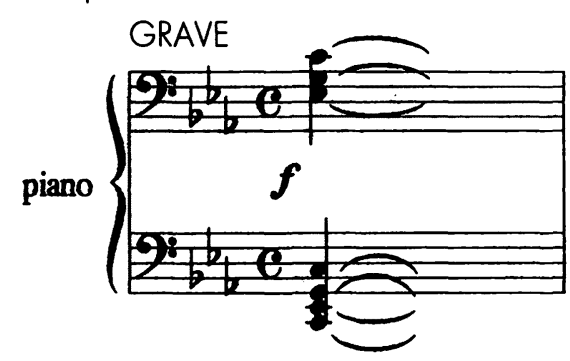

$\mathrm{Si}$, un court instant, tout le climat de la Sonate pathétique est créé, ce n'est sûrement pas parce que Beethoven a écrit un accord de do mineur, mais à cause du formidable - j'allais dire tragique - brouillage non écrit mais entendu, au centre le plus sensible de la perception auditive, c'est-à-dire dans le registre moyen ébranlé par l'accord grave.

Nous entendons, en plus de l'accord écrit, tous ces sons virtuels là partir des trois notes les plus graves de l'accordl:

Exemple 2

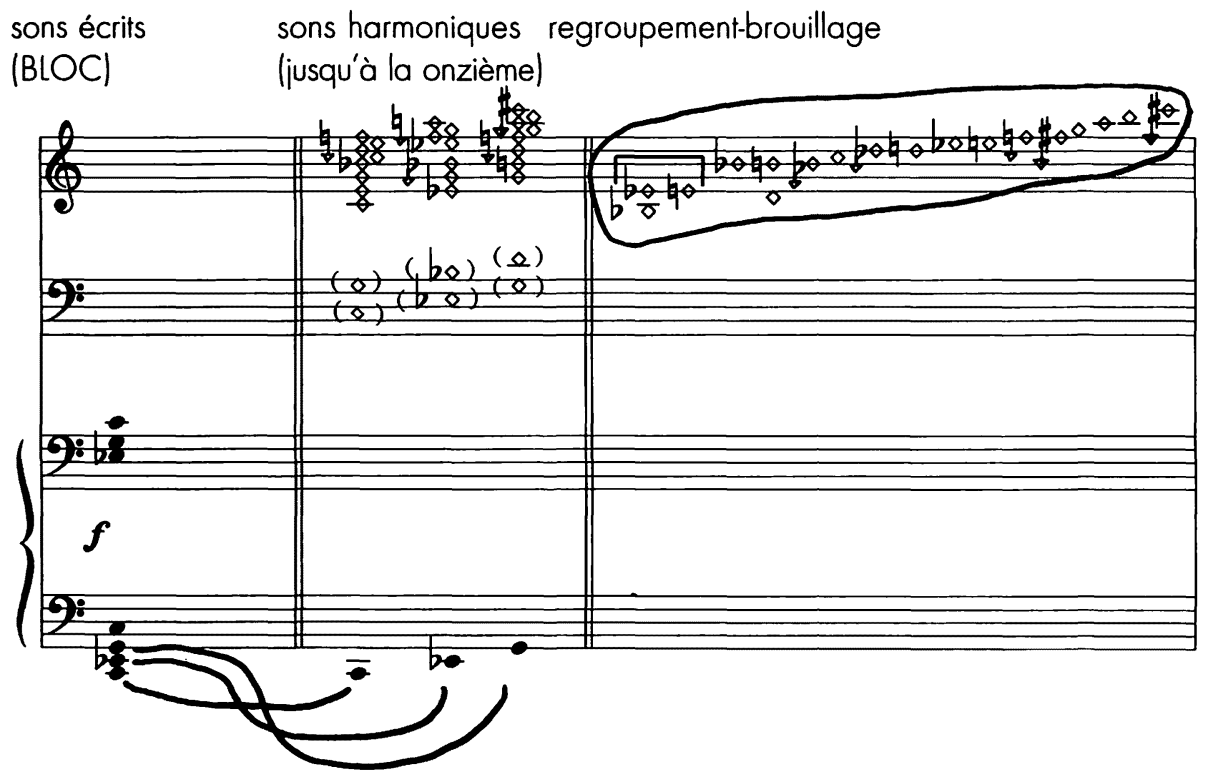


Donc un réseau principal issu du do grave et deux réseaux qui le contrarient ou l'appuient partiellement, celui du mi bémol et celui du sol bécarre, celui du mi bémol étant le plus perturbateur, notamment par la friction mineure-majeure entre mi bémol (quatrième harmonique de mi bémol) et mi bécarre (cinquième harmonique de dol.

Nous venons de nous attacher à la réalité du phénomène sonore, à l'exemple de Varèse qui, à l'issue d'une de mes premières visites, me dit en substance : «Noublions pas que, pour nous musiciens, c'est le son qui est l'un de nos meilleurs maîtres. C'est pourquoi il faut l'observer et l'étudier, ses leçons sont inépuisables. »

Or le son contient une complexité croissante d'harmoniques aiguës : crescendo du complexe. Varèse les soulignera ou les contredira, en les intensifiant par un travail des dynamiques et des rythmes. II en recherchera les confins harmoniques les plus lointains, comme attiré par les régions de l'inaudible, à l'image de la spirale qui le fascinait tant, giration qui ne s'arrête jamais :

\section{(...) J'irai par-delà le visible}

\section{Même le dernier mot recule(1).}

II en résultera, sous formes de spectres (donc de timbres) nouveaux, une musique aimantée par le dépassement des sevils, en une exploration qui, à l'image même de la perception du son et de ses harmoniques le plus lointaines, contient déjà une dynamique formelle de croissante complexité, mouvement que l'on retrouve également au plan des intensités dans une des courbes dynamiques types de Varèse ${ }^{(2)}$, un crescendo qui ne s'arrête que par l'impossibilité d'aller plus loin.
(1) OElenschlager, poète danois cité par Varèse à la fin de la conférence de Princeton, 4 septembre 1959. Traduit dans liberté $59, n^{\circ} 5$, Montréal.

(2) Messiaen a comparé ce type de crescendo à une résonance rétrogradée. 
Exemple 3
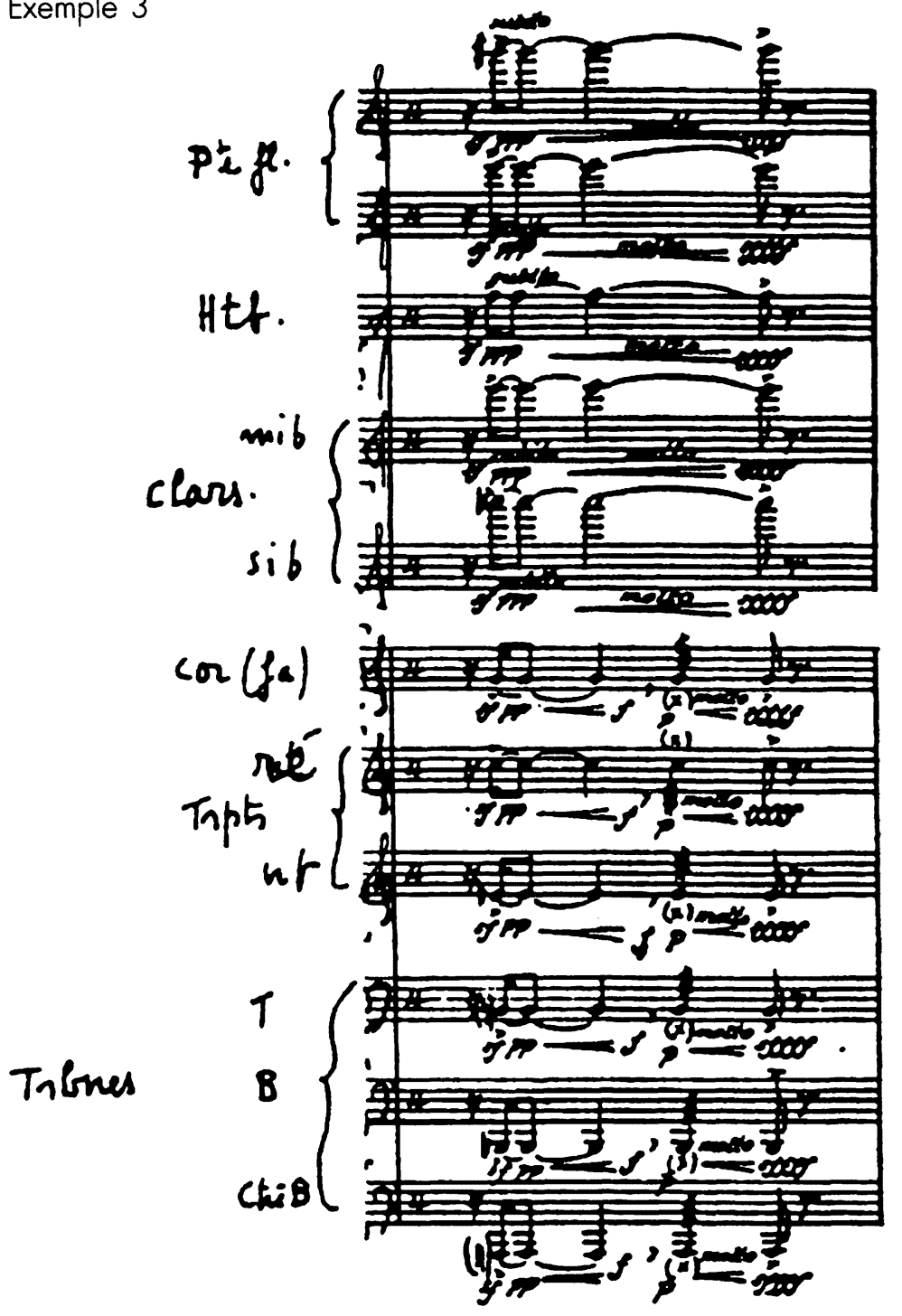

Intégrales, p. 18.

Dans ces blocs sonores, qui sont un des aspects les plus typiques de son langage, Varèse utilisera deux principes: 1/ celui des sons harmoniques naturels et des sons résultants, provoquant entre les sons renforcement réciproque de l'intensité, détente, stabilité ; 2) et celui de fréquences étrangères engendrant, par leurs propres réseaux, perturbation, tension, déstabilisation. 
Ces réseaux se croiseront, se repousseront ou s'attireront, branches contraires organiquement liées les unes aux autres. Autrement dit, plus il y aura corrélation avec les harmoniques, plus le bloc sera stable; et le contraire: moins il y aura de corrélation, plus le bloc sera instable. Selon ce schéma:

Disposition naturelle AVEC

(la série harmonique) renforcement de l'intensité

Résultat stabilité

détente

\section{CONTRE}

(la série harmonique) perturbation

instabilité

tension

\section{NOTE}

Ce principe a des conséquences qui peuvent paraître surprenantes. Ainsi, un même intervalle classé comme "consonant " deviendra " dissonant " (relativement) selon son registre.

\section{Exemple 4}

Brouillage à cause des harmoniques

tierce

mineure

grave

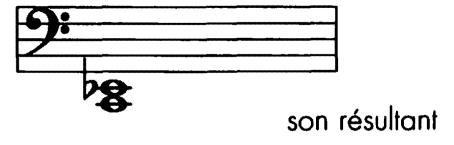

ou sa disposition.

Tension-instabilité (x dissonant $» \mid$ sans rapport harmonique
Clarté : on ne perçoit presque pas les harmoniques, et l'intervalle est unifié par le son résultant : la bémol

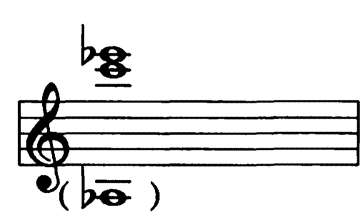

\section{tierce}

mineure

aigüe

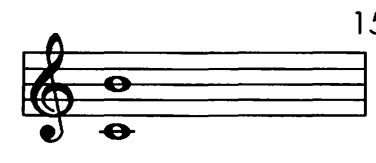

Détente-stabilité

(a consonant $»$ ) avec rapport harmonique $1-15$

$15^{\circ}$ harmonique

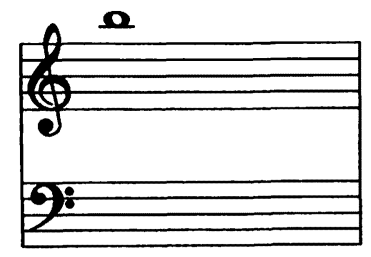

fondamentale 
Pour revenir au rapport entre le registre et le brouillage-tension, on peut dire qu'un même complexe sonore aura une tension par brouillage harmonique croissant vers le grave et décroissant vers l'aigu. Notre même accord-bloc de Beethoven n'a plus du tout la même nature si on le transpose deux octaves plus haut, pour la bonne raison que l'on ne perçoit que les harmoniques provenant du grave, alors que ceux du registre aigu ne sont presque pas perçus, aux limites de l'audible, ce qui enlève au complexe l'impression de brouillage et d'épaisseur. Ce sont exactement les mêmes notes, les mêmes intervalles, le mệme accord. En apparence. La personnalité a changé. En réalité, il ne s'agit plus du même complexe.

L'exemple de Beethoven était non concordant. En voici un au contraire où toutes les fréquences sont à leurs places dans la série harmonique. (Pelléas, acte I, scène I).

Exemple 5

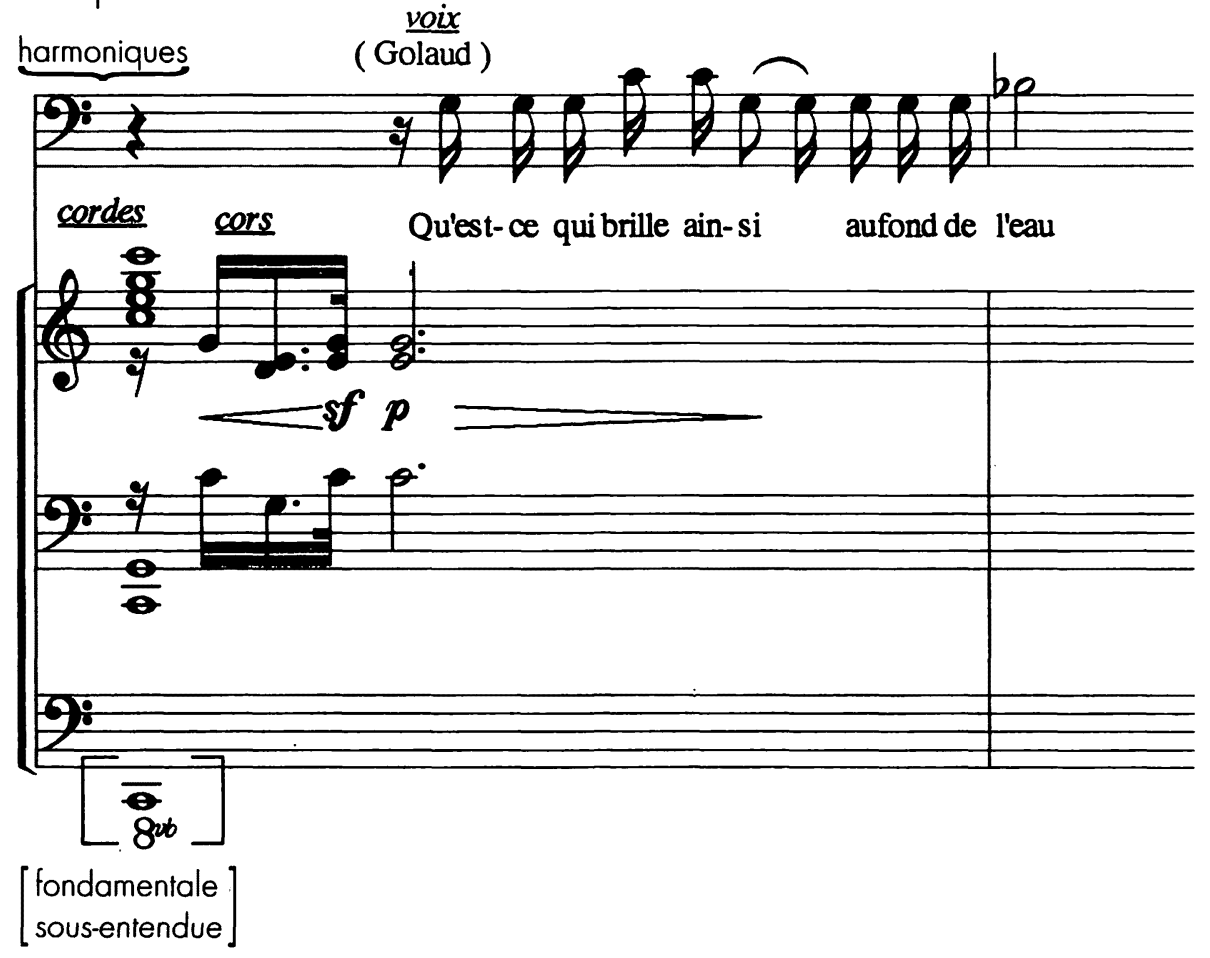

Debussy, Pelléas et Mélisande, acte I, scène I.

(Le si bémol n'est doublé par aucun instrument. Debussy a ainsi voulu permettre à la voix de chanter un véritable septième harmonique, donc à intonation plus basse que le si bémol.) 
A la définition de la musique d'Hoene Wronski (savant et philosophe du xixe siècle) que Varèse aimait citer \& la corporification de l'intelligence qui est dans les sons \#, j'associerai celle-ci : la musique est \& une science physico-mathématique; le son en est l'objet physique, et les rapports trouvés entre les différents sons en font l'objet mathématique ». Elle pourrait être de Varèse, elle est de Rameau ${ }^{(3)}$ !

Trouver l'« intelligence qui est dans les sons », n'est-ce pas prendre conscience des divers courants qui les traversent? Amorcée avec Beethoven, Debussy, Rameau, c'est cette démarche même du compositeur qui nous guide. Varèse n'aurait point récusé ces compagnons d'exploration.

\section{blocs :}

Voici donc, à partir de ces critères de résonance, l'analyse de quelques

1) la fin d'Hyperprisme qui est également le point d'aboutissement et le moment de radiation maximum de l'œuvre, où se retrouve le total chromatique ainsi échelonné :

Exemple 6
(3) *Traité de l'harmonie in GIRDLESTONE, Cuthbert (1962), p. 521.

(4) A cause du contexte, c'est le do qui était la note la plus centrale mélodiquement et pour l'oreille. C'est donc elle qui est la plus affectée par l'éclat radiant qui l'entoure dont elle tient cependant la crête la plus aiguë, le sol sifflant, point de jonction commun entre les forces contraires. Remarquons que le mi qui est la note la plus isolée de l'ensemble a cependant une relation voisine relativement simple (5/4) qui privilégie sa parenté au do. Sa troisième harmonique est un si, ef le si est la note la plus grave du complexe.

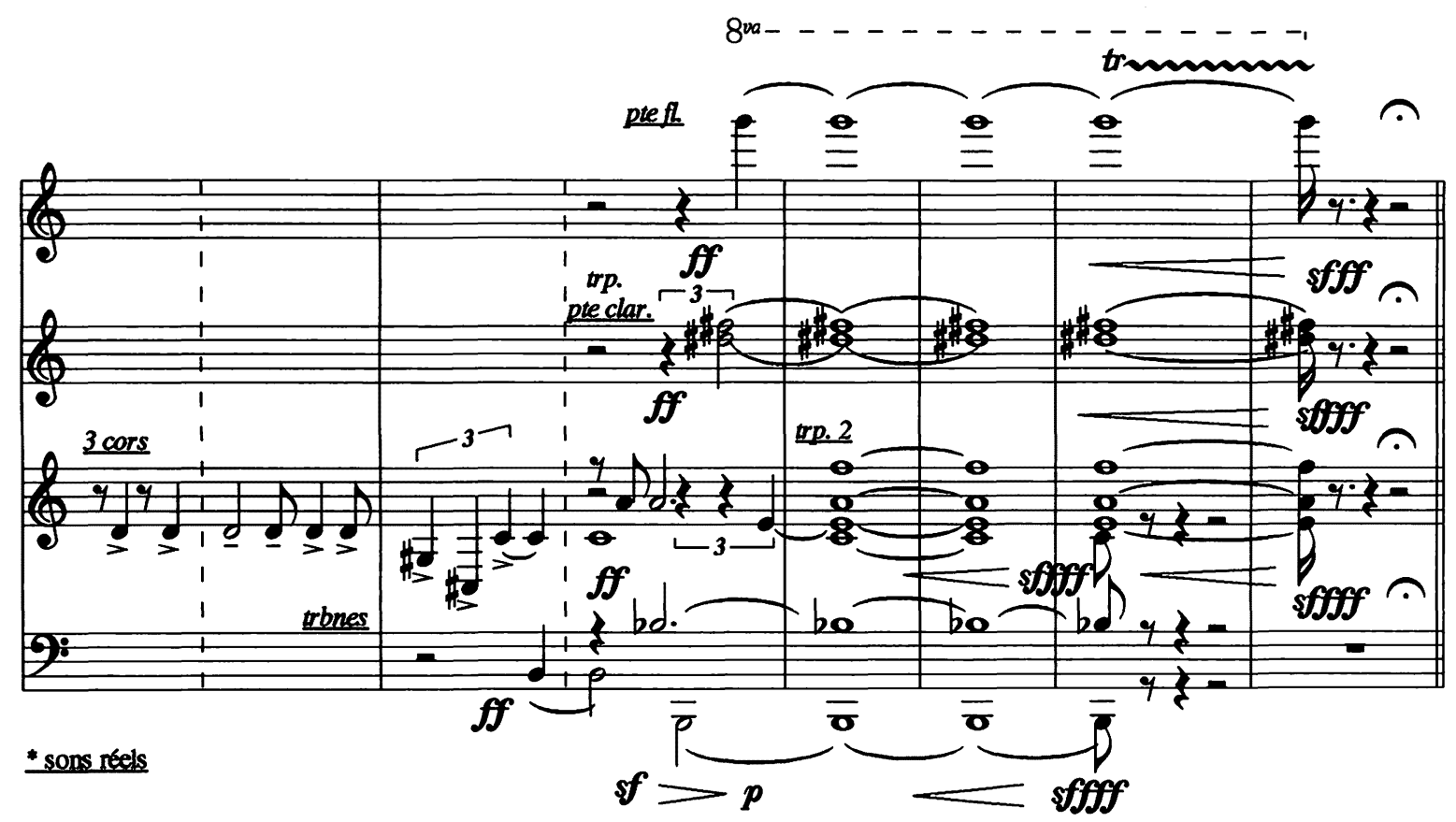

Hyperprisme $(\text { fin })^{(4)}$. 
soit :

détente

(renforcement de l'intensité)

tension (perturbation)

Exemple 7

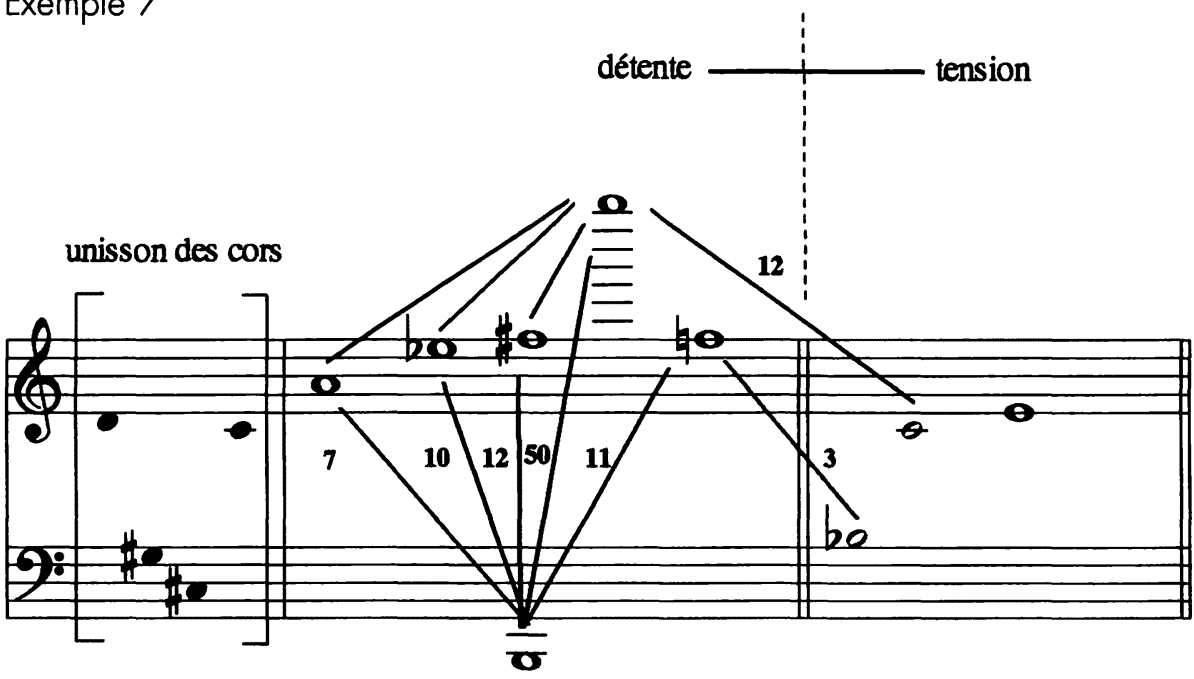

total chromatique

Exemple 8

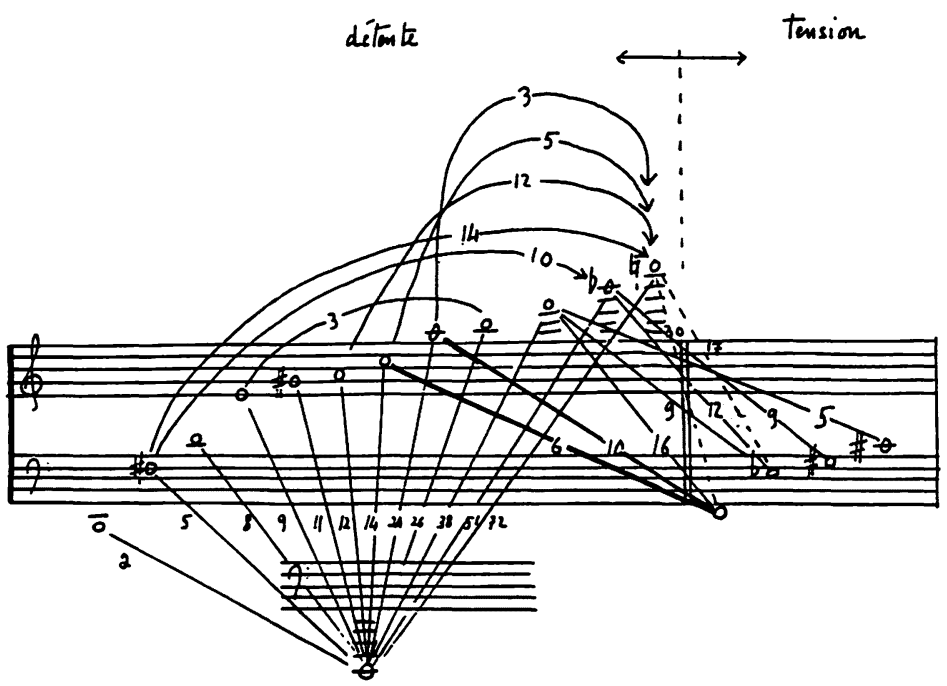

Amériques (dernier bloc harmonique). 
Dans la première partie d'Intégrales, les trois blocs harmoniques sont scindés en couches indépendantes ayant chacune leurs propres dynamiques. Is sont composés de formants acoustiques soit en opposition (par tensions plus ou moins grandes), soit en éclat.

Exemple 9

$-\longleftrightarrow$ tension $\longrightarrow+$

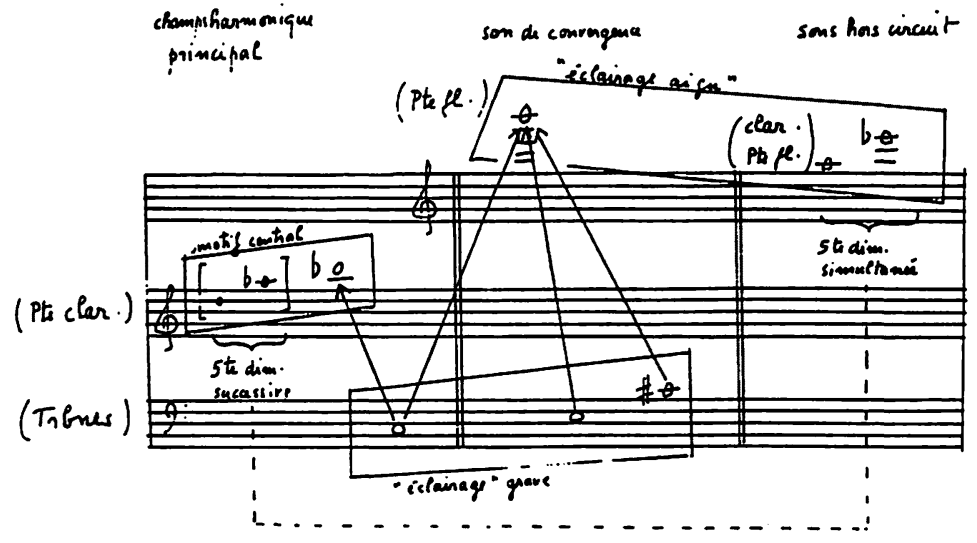

Intégrales, «Bloc I»,pp. 1 à 7.

Exemple 10 SYMÉTRIE II

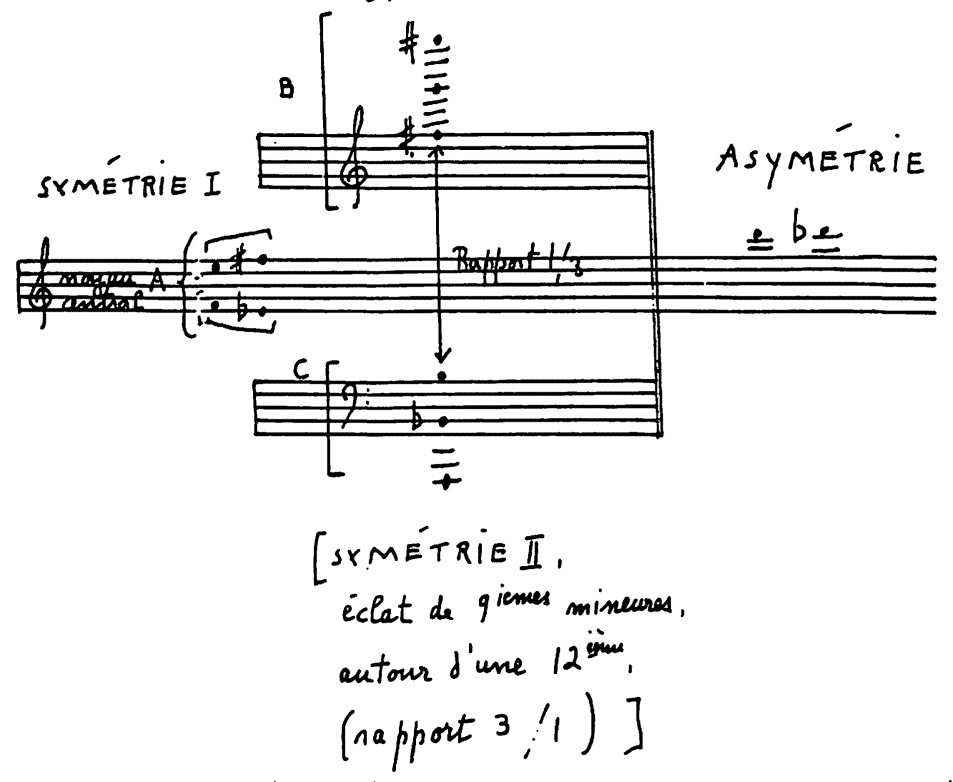

Intégrales, «Bloc II », p. 8. Symétrie et asymétrie, éclat. 
Exemple 11

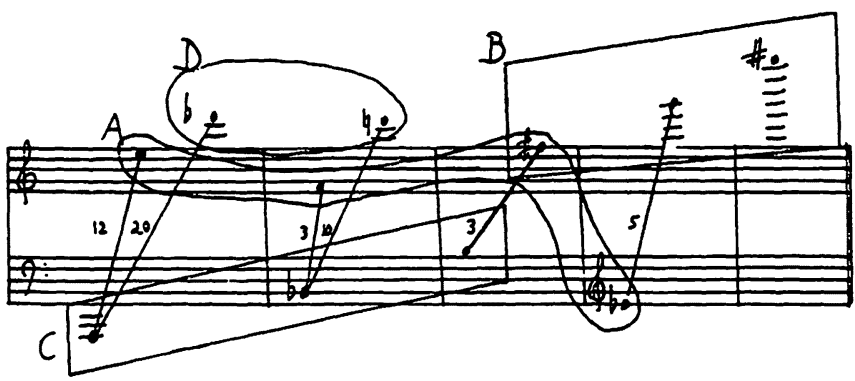

Intégrales, "Bloc II », en éclat harmonique.

$C, B$ : symétriques.

$A, D$ : asymétriques (par rapport à l'ensemble).

Si l'on effectuait une symétrie de $B$ au rebord inférieur de $A$, on aurait mi bémol, ré bécarre, ré bémol. Ce sont ces mêmes notes qui servent à l'asymétrie.

Dans ce deuxième bloc, il n'y a pas de grand réseau harmonique prioritaire sinon l'axe si-fa dièse (1-3), qui réunit les éclats des extrêmes:

Exemple 12

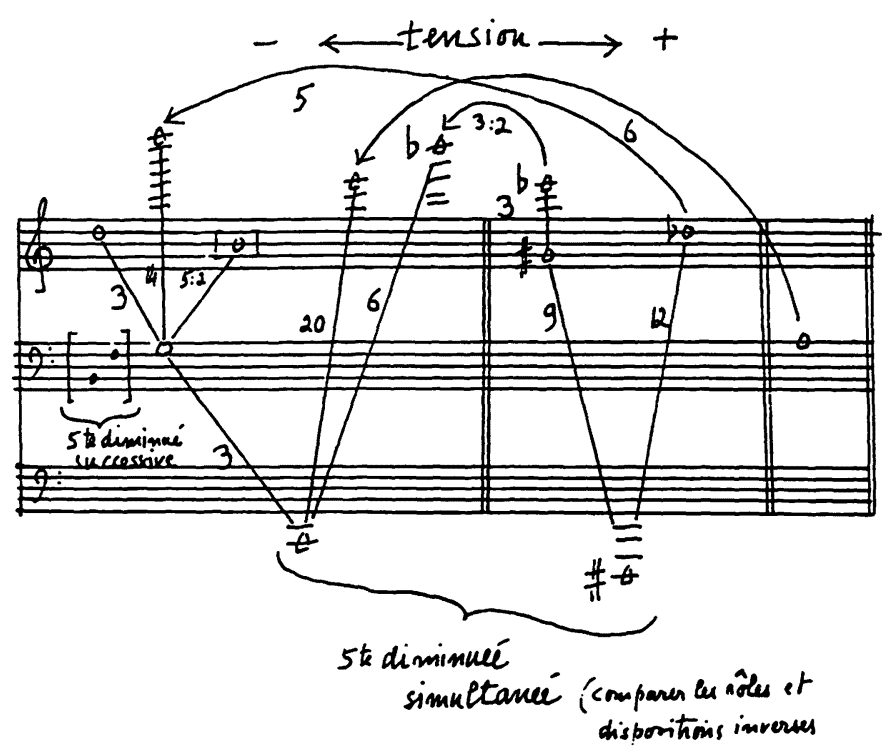

Intégrales, Bloc III, pp. 9 à 14, «Transmutation du Bloc 1», Re-formation. 
Rapport du formant des trois trombones (Bloc I) avec le formant deux trompettes-trombone (Bloc III).

Exemple 13
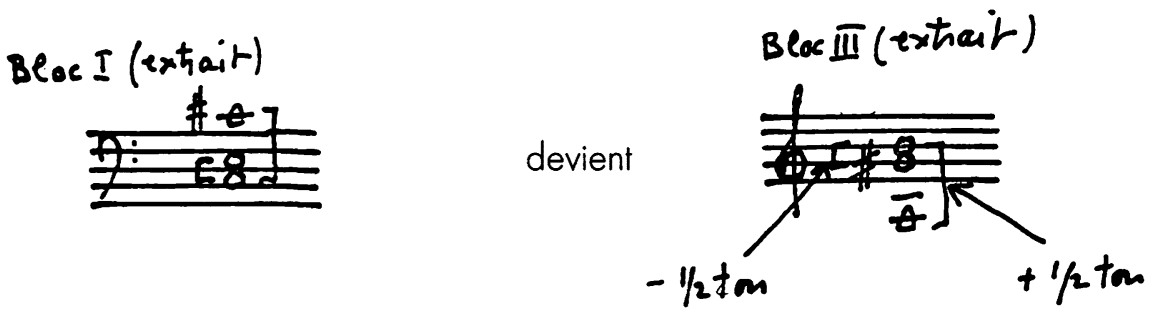

«Transmutation » par renversement, agrandissement (neuvième) et rétrécissement (tierce) des intervalles.

À partir de ces coups de sonde, quelques constatations s'imposent :

1. La qualité de l'éclat, à sonorité maximale, due à la solidarité des différentes composantes sonores, qui se renforcent mutuellement par leurs courants harmoniques, mis en relief par une tension extrême, acérée, "évidence acoustique » assurant la netteté de l'articulation du discours musical. (Boulez, 1991, p. 3661

2. À la progression du spectre sonore, au formant de plus en plus complexe et ramifié vers l'aigu, correspondent une dynamique exploratoire, une morphologie de la croissance.

3. Par déduction, la forme sera également formation à l'image de la naissance du son, de sa genèse, construction de formants en échafaudages successifs. Je pense en particulier à la quinte à vide qui marque l'entrée des voix de basses dans Ecuatorial. Toute l'œuvre semble être fondée sur cette quinte - le premier des intervalles - à la fois faisceau convergent et rayonnement de l'œuvre. II est d'ailleurs significatif, sinon symbolique, que les premières paroles chantées à partir de cette quinte soient : "ô constructeurs, ô formateurs, vous voyez, vous entendez... "(tiré du Popol Vuh Maya-Quiché). Le musicien-acousticien se trouve ici en parfaite correspondance avec l'innovation de la « tribu».

4. La complexité même des blocs sonores, afin qu'ils soient pleinement perçus, nécessite la durée, la réitération, donc le statisme ${ }^{(5)}$. Cette nécessité d'imprégnation donne naissance à des formes litaniques en incantation, par phases amples et inégales, toujours variées, en un mouvement qui, étant exclu des fréquences, envahit toutes les autres composantes de la musique. Curieusement, une des musiques les plus apparentées à Varèse à cet égard
(5) Encore Rameau : « plus la même harmonie a de durée, plus elle a le temps de pénétrer jusqu'à l'âme et de l'affecter au point qu'elle s'est proposé *. Cité in GIRDLESTONE (1962), p. 509. 
est peut-être celle des rites bouddhiques du Tibet, avec ses incantations, ses trompes aux dimensions impressionnantes, ponctuées par des éclats de métaux ou de peaux aux décalages variés.

Varèse, au moment où l'écrivain Fernand Ouellette lui fit écouter l'enregistrement de l'expédition de Serge Bourguignon (1955), n'avait jamais entendu de musique tibétaine.

Du rapport entre blocs et monodies, on peut tirer une loi : plus le spectre est complexe (blocs), plus le mouvement est d'ordre dynamique lagogique de l'intensitél ; plus le spectre est simple, plus il est d'ordre mélodique lagogique des fréquences). Ainsi, les soli d'Octandre ou d'Intégrales seront libres de toute entrave harmonique. Lorsque le spectre se rebâtira en blocs sonores, les fréquences redeviennent statiques - à divers degrés, cela va de soi. On peut même observer ce phénomène dans une œuvre tout à fait monodique comme Densité 21,5 pour flûte seule. La mélodie y est essentiellement évoluante avec ses progressions chromatiques. Par contre, aussitôt qu'elle est pensée harmoniquement, elle devient plus statique, comme dans ce passage, sur un nombre limité de trois notes.

Exemple 14<smiles>CC(C)=[Te]</smiles>

(flate)
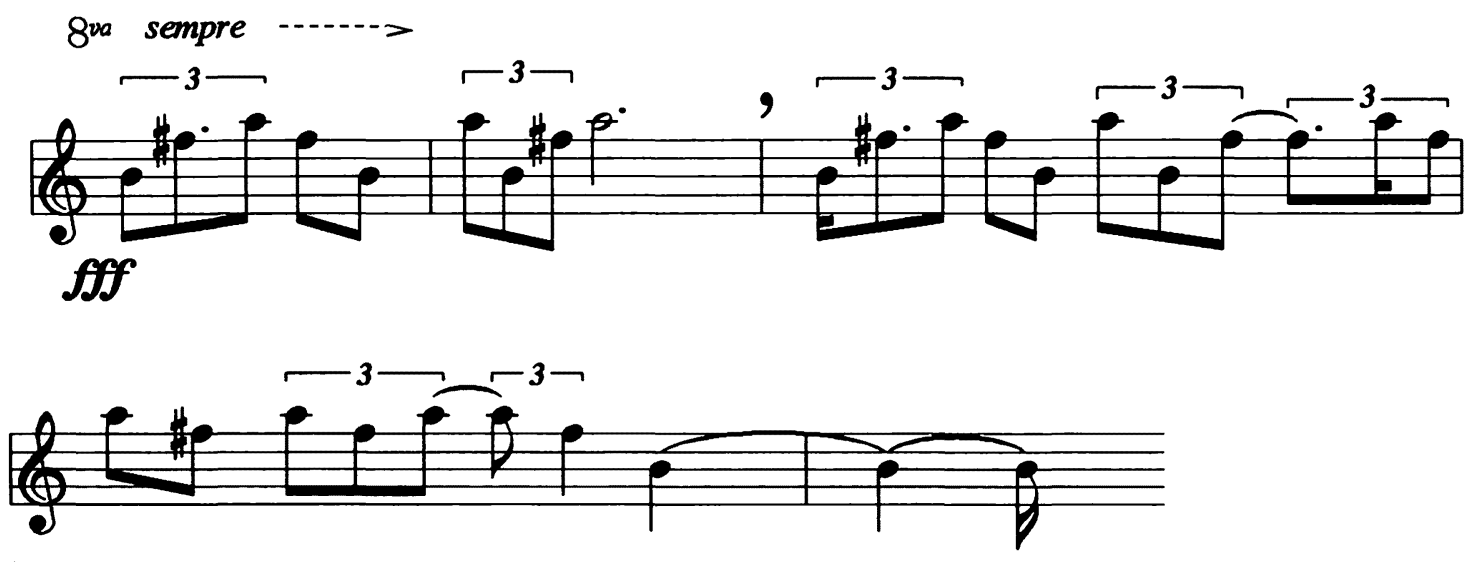

Densité 21,5. Statisme dans la mélodie.

On trouvera cependant des exceptions à cette règle, comme au début d'Amériques, où les rôles sont inversés : la flûte répète la même cellule, et les interpolations de plus en plus importantes de l'orchestre varient et envahissent la musique. Exemple: les dix-huit premières mesures d'Amériques. 
Il est intéressant de noter dans les rapports bloc vertical et monodie horizontale l'adéquation remarquable des deux axes. Le matériau est équivalent. Le simultané couvrant un registre large devient successif à l'intérieur d'un registre restreint (voir le solo de hautbois d'Intégrales par rapport à tous les grands blocs de l'œuvre).

Si les blocs harmoniques sont une des caractéristiques de l'œuvre de Varèse, y circonscrire la relation "acoustique ef forme " l'amputerait d'aspects importants.

Parmi ceux-ci, il faut mentionner entre autres:

1. La relation hauteur-tempo. La vitesse de vibration des fréquences trouvant son équivalent dans le tempo ${ }^{(6)}$, technique largement exploitée dans Déserts, surtout à partir de la deuxième séquence d'orchestre, ainsi que dans l'interpolation de qui suit, au moyen d'une cellule de notes répétées dont les vitesses extrêmes sont :

Exemple 15

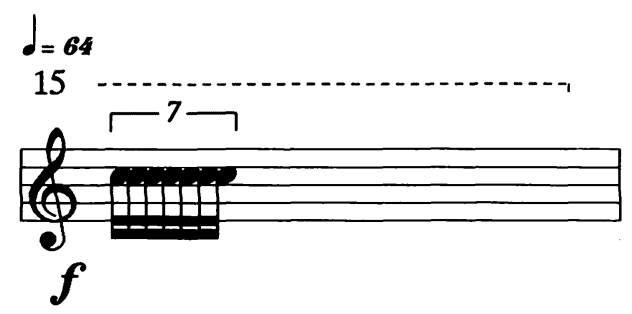

au xylophone

et à la timbale

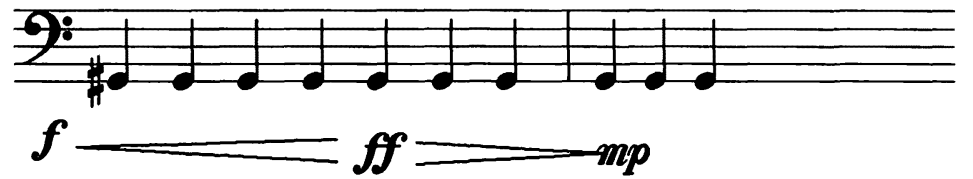

Déserts, mesure 57 et suivantes.

Entre ces extrêmes, on peut suivre la cellule qui subit de multiples transformations et variantes, notamment dans les sons du xylophone ralentis de deux octaves, à la caisse claire, au cor, etc.

2. La morphologie phonétique (c'est-à-dire la forme des attaques, les profils de timbre et d'intensité). Cet aspect à lui seul nécessiterait une étude poussée. En voici les grandes lignes:

1) I'influence des percussions auprès des instruments de l'orchestre: rythmes sur une seule note, en général aux peaux aiguës ou aux blocs de bois /style insecte...), que l'on retrouve aux instruments à sons tenus ;

2) les profils travaillés des résonances métalliques, droits $(>)$ ou contraires $|<|$ ou couches à dynamies multiples et indépendantes (contrepoints d'intensités);
(6) A l'instar de Mozart et Berlioz. Mo zart, comme le fit remarquer Stockhausen lors de ses cours à Darmstadt, avait eu cette intuition. Lorsqu'il module à la dominante, on a très souvent l'apparition de triolets, ce qui donne une relation 3 pour 2 , comme la quinte. Berlioz, dans le final de la Symphonie fantastique, fait correspondre registre et durée de façon spectaculaire, en trois étages successifs, lors de l'apparition du Dies Iræ (dernier mouvement, mesure 127): thème en valeurs longues et diminution de moitié, puis du quart, selon les octaves. 
3) phénomène de contagion comme celui du tambour à corde (lion's roar) faisant écho au hurlement d'orchestre.

Le statisme de Varèse mérite réflexion : il n'avait jamais été poussé aussi loin avant lui. J'y vois une véritable poétique du stable et de l'instable. Le stable contenant toujours son contraire le mène souvent au bord de l'instable. II suffit alors d'un événement minime (un nouveau son ou une note déplacée) pour le faire basculer en son contraire. Cela s'effectue toujours après un laps de temps assez long, incantatoire, laissant le temps à l'état de durée, mais soumis à une sorte d'érosion (par le travail des dynamies et du rythme) où l'oreille a le loisir de balayer le spectre sonore et d'en saisir la complexité et les courants contraires (je dirais presque l'aventure potentielle). Un événement étranger survient, et tout se désintègre pour se re-former autrement. Ce scénario n'est pas celui d'une coupe inéluctable ou déterminée, mais celui d'un processus formel, le déroulement dans le temps n'étant jamais aussi simple, avec ses multiples tropes, ses développements souterrains (Hyperprisme), montagnes submergées dont les crêtes surgissent comme des signes géographiques inattendus dans la fantaisie des îles; où l'état (le statisme) des êtres sonores contient le germe du mouvement, virtualité de formes.

De cette réflexion sur « acoustique et forme», je retiendrai essentiellement deux idées conjointes:

- une dynamique exploratoire au-delà du perceptible, donc ascendante, faisant reculer les limites, croissances en ondes « cristallisantes », par vagues successives comme le sont les ondes sonores, litanie ondulatoire ;

- et, conjuguée à cette dynamique, une poétique du stable et de l'instable, d'un stable sans cesse déstabilisé, d'état en état. Image de la création.

Exemplarité.

Ce premier centenaire est aujourd'hui l'occasion d'en célébrer le legs.

BOULEZ, Pierre (1981), Points de repère, Paris, Christian Bourgois éditeur.

GIRDLESTONE, Cuthbert (1962), Jean-Philippe Rameau, sa vie, son œuvre, Bruxelles, Desclée de Brouwer.

Musique tibétaine du Sikkim, 1955. Contrepoint (30) M.C. 20.119, collection du Musée de l'homme. 


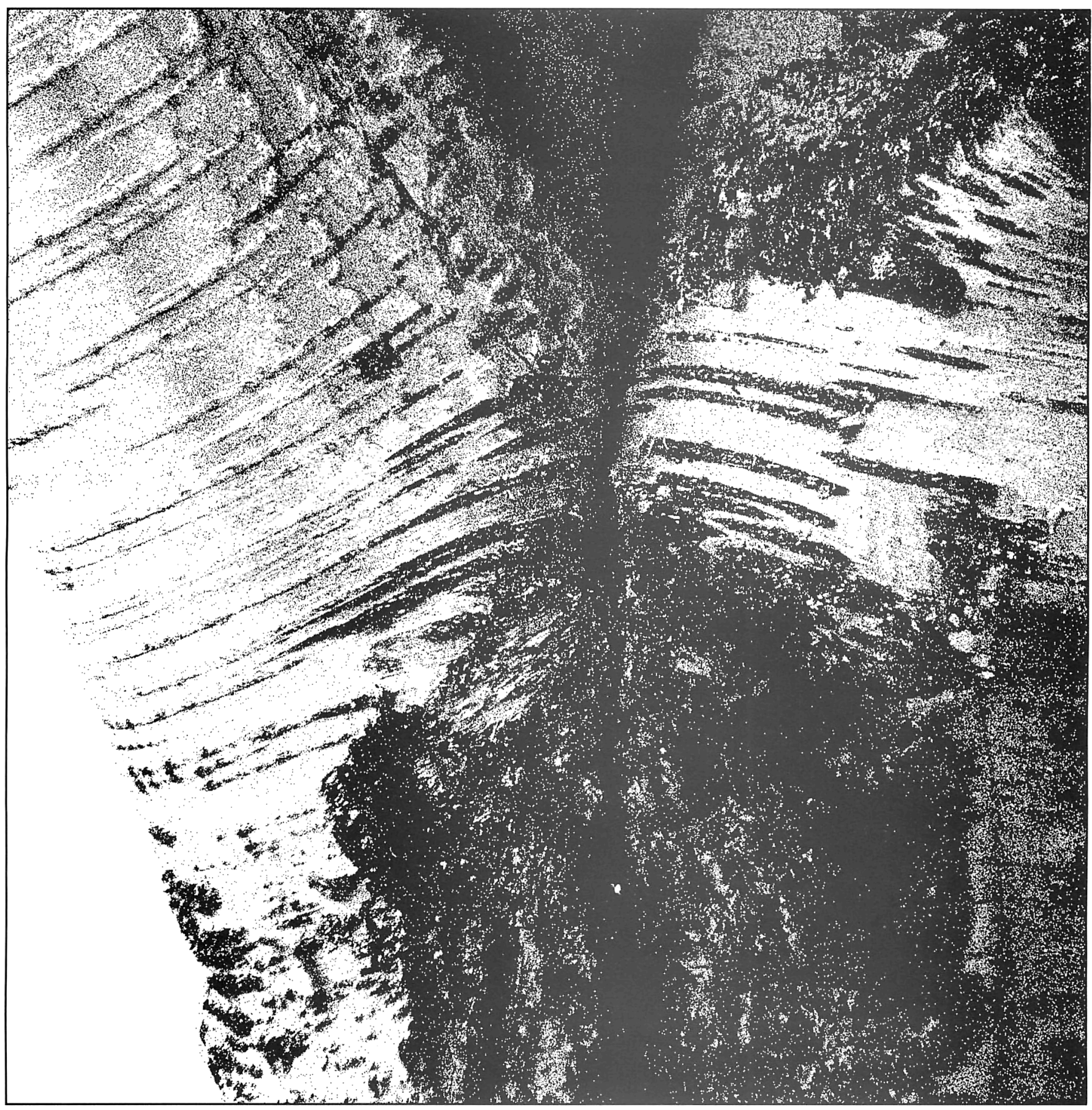

